\title{
Some practical crack path examples
}

( da ESIS Newsletter 2006)

\author{
Les P. Pook \\ Department of Mechanical Engineering, University College London, Torrington Place, London WC1E 7JE, \\ UK
}

\begin{abstract}
It is well known that many engineering structures and components, as well as consumer items, contain cracks or crack-like flaws. It is widely recognised that crack growth must be considered both in design and in the analysis of failures. The complete solution of a crack growth problem includes determination of the crack path. Macroscopic aspects of crack paths have been of industrial interest for a very long time. At the present state of the art the factors controlling the path taken by a crack are not completely understood. Eight brief case studies are presented. These are taken from the author's professional and personal experience of macroscopic crack paths over many years. They have been chosen to illustrate various aspects of crack paths. One example is in a component from a major structure, three examples are in laboratory specimens, and four are in nuisance failures. Such nuisance failures cause, in total, a great deal of inconvenience and expensive, but do not normally receive much publicity.
\end{abstract}

KEYWORDS: Crack growth, crack path

\section{INTRODUZIONE}

It is well-known that engineering structures and components, as well as consumer items, contain cracks or flaws and, therefore, crack growth must be considered both in design and in the analysis of failures. The path taken by a crack in a critical component or structure can determine whether fatigue failure is catastrophic or not. Knowledge of potential crack paths is also needed for the selection of appropriate non-destructive testing procedures. Much current work is concerned with crack growth viewed on macroscopic scale. The forthcoming ESIS International Conference on Crack Paths (CP 2006) will be devoted to consideration of crack paths at various scales. From a theoretical viewpoint the complete solution of a crack growth problem includes determination of the crack path.

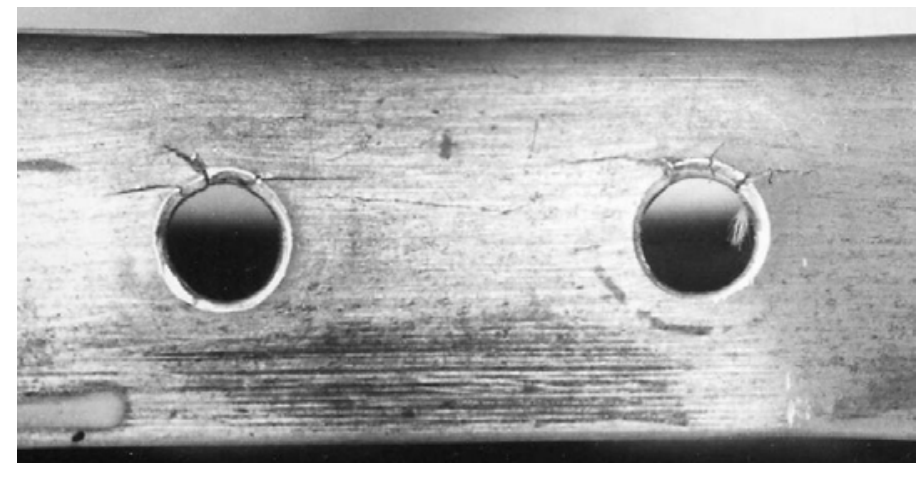

Figure 1: Cracks in undercarriage bay bracket.

It is often assumed that the crack path is known, either from theoretical considerations, or from the results of laboratory tests. At the present state of the art, the factors controlling the path taken by a crack are not completely understood [1]. Eight brief case studies involving crack paths are presented. These are taken from the author's professional and personal experience over many years.

They have been chosen to illustrate various aspects of crack paths. One example is in a component from a major structure, three examples are in laboratory specimens, and four are in nuisance failures. Such nuisance failures cause, in total, a great deal of inconvenience and expensive, but do not normally receive much publicity.

\section{AIRCRAFT UNDERCARRIAGE BAY BRACKET}

The relationship between mode of fatigue loading and paths taken by fatigue cracks has been of interest for a long time [2, 3]. This information can be useful in failure analysis and Figure 1 shows an example from 1961. It is a bracket from an aircraft undercarriage bay which showed unexpected cracking at rivet holes. The bracket was a formed $18 \mathrm{swg}$ (1.2 mm thick) aluminium alloy angle, 10 in $\times 0.8$ in $\times 0.8$ in $(254 \mathrm{~mm} \times 20.3 \mathrm{~mm} \times 20$. 3 $\mathrm{mm})$. The figure shows a general view of typical cracks observed after the bracket was removed from the bay. Examination of the fracture surfaces of the cracks showed that fatigue cracks had originated at both surfaces of the bracket at the rivet hole corner and then propagated inwards on elliptical crack fronts, with the two cracks intersecting at or near the centre line of the sheet. Figure 2 shows the fracture surface of a typical crack. This indicates that failure was caused by out of plane alternating 
bending fatigue loads, which were not anticipated by the designer. Examination of the fracture surfaces at high magnification showed the presence of striations and hence confirmed that cracking was due to fatigue. This is an example of the useful crack path information which can be obtained from simple examination of a failed component with the naked eye.

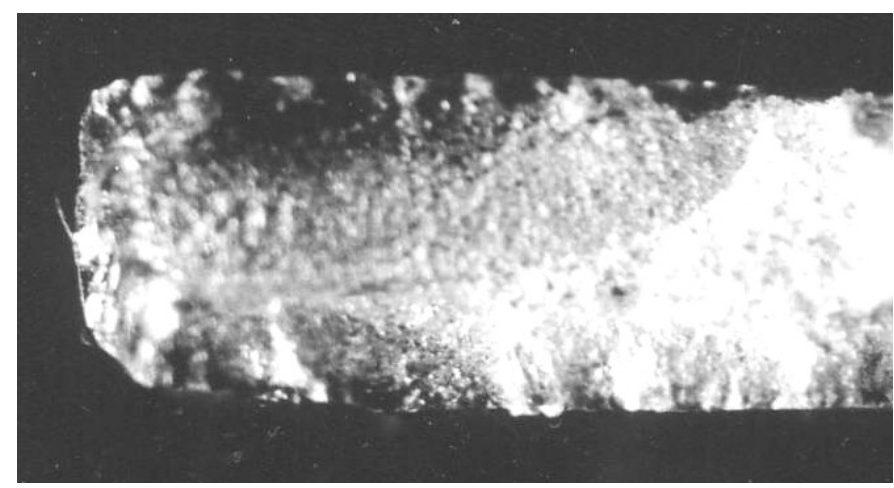

Figure 2. Surface of crack in an undercarriage bay bracket.

\section{ANGLE NOTCH FRACTURE TOUGHNESS AND FATIGUE SPECIMENS}

By 1965 plane strain fracture toughness testing using Mode I specimens, in which crack growth is perpendicular to the applied load, was well established [4] but little was known about fracture toughness behaviour under mixed mode loading, where loads are applied at an angle to the crack.. Some tests were therefore carried out in $1966[5,6]$ to investigate the mixed mode fracture toughness of DTD 5050, a $5 \frac{1}{2} \% \mathrm{Zn}$ aluminium alloy with $K_{\mathrm{Ic}}=$ 28.8 $\mathrm{MPa} \sqrt{\mathrm{m}}$ [5]. A $19 \mathrm{~mm}$ thick angle notch specimen was used, with the initial notch inclined at an angle $\beta$ of $75^{\circ}, 60^{\circ}$ and $45^{\circ}$, as in Figure 3 . Specimens were precracked in fatigue. Figure 4 shows the fracture surface of one of the specimens with the initial notch inclined at $\beta=$ $45^{\circ}$. The fatigue precrack (bright area at the notch root) is of nearly constant depth, and at the end of the precrack $\beta$ $\approx 48^{\circ}$. A feature of the test is that under the static loading to determine the fracture toughness the specimen failed very abruptly, but the macroscopic crack path features followed on from the fatigue precrack. At the time the fracture surface appearance was puzzling, but is easily interpreted from a modern viewpoint [1], in that that there is a tendency to Mode I crack growth on two scales. On a scale of $1 \mathrm{~mm}$ initially crack growth was mixed mode. As the crack grew the crack front rotated until it was perpendicular to the specimen surfaces, and crack growth was in Mode I, with the exception of shear lips at specimen surfaces. On this scale the crack follows a curved path which tends towards a plane of symmetry. This is in accordance with the well known observation [1] that the tendency to Mode I crack growth means that cracks tend to grow perpendicular to the maximum principal tensile stress. On a smaller scale of $0.1 \mathrm{~mm}$ the tendency to Mode I fatigue crack growth results in the production of what is known as a twist crack [1] containing individual Mode I facets connected by cliffs. The Mode I facets gradually merge as, viewed on the $1 \mathrm{~mm}$ scale, the crack growth surface becomes perpendicular to the specimen surfaces. Merging of Mode I facets shows up more clearly under fatigue loading.

Some fatigue tests were carried out in 1989 on $20 \mathrm{~mm}$ thick medium strength structural steel angle notch specimens [7] with initial $\beta$ values of $75^{\circ}, 60^{\circ}$ and $45^{\circ}$. Figure 5 shows the fracture surface of one of the specimens, initial $\beta=60^{\circ}$. The light area at the top is where the specimen was broken open in liquid nitrogen. These examples illustrate the strong tendency to Mode I crack growth in isotropic materials under essentiallv elastic conditions.

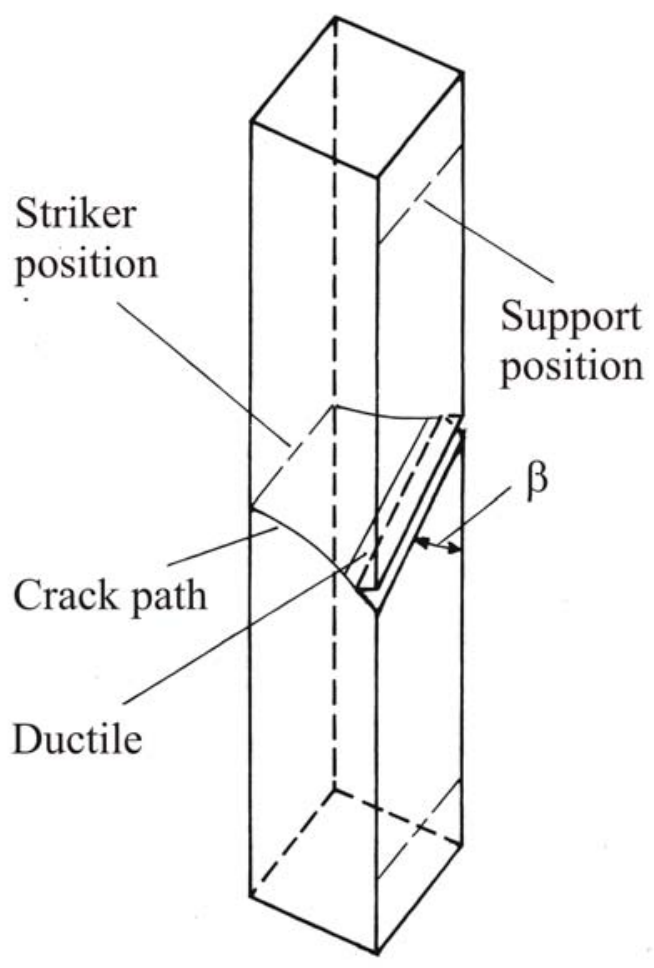

Figure 3. Angle notch Charpy specimen, crack initiation along notch tip.

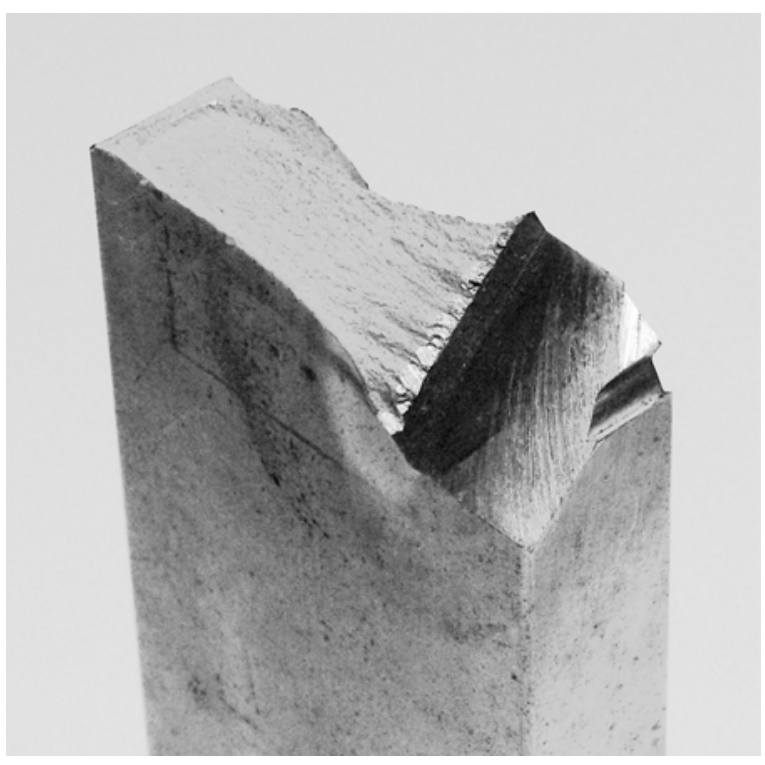

Figure 4. Fracture surface of DTD 5050 51ำ Zn aluminium angle notch fracture toughness test specimen, initial $\beta=45^{\circ}$. 


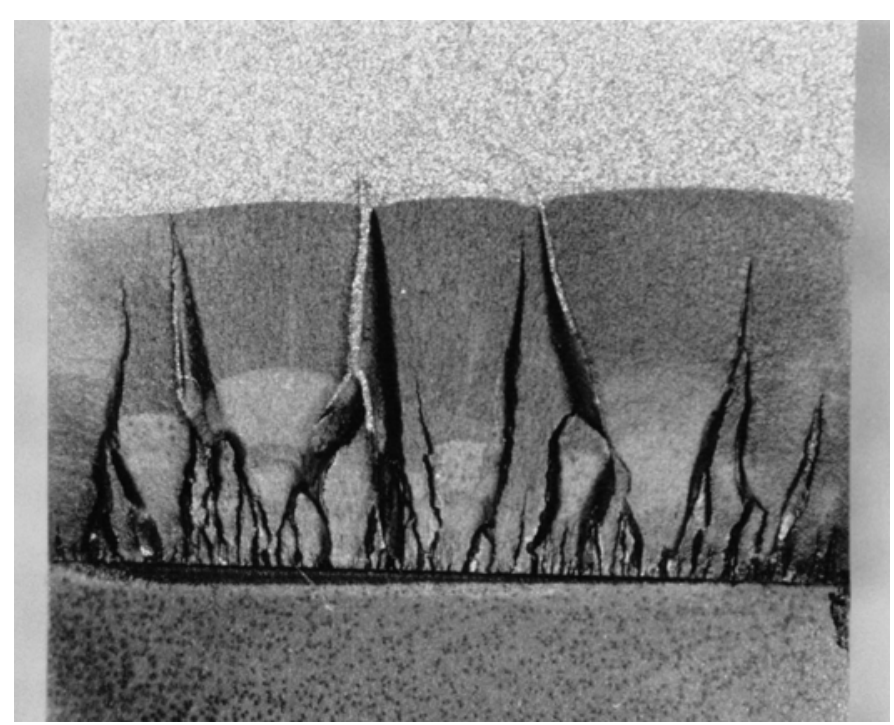

Figure 5. Fracture surface of medium strength structural steel angle notch fatigue test specimen, initial $\beta=60^{\circ}$.

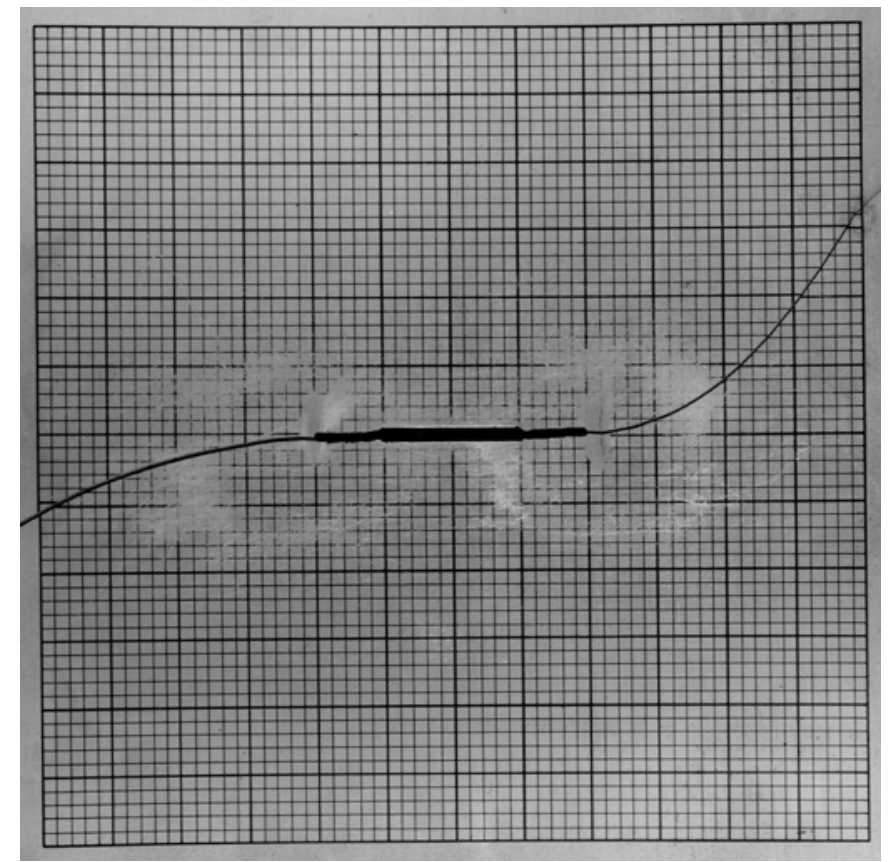

Figure 6. Crack path in a Waspaloy sheet under biaxial fatigue load. The grid is $2.54 \mathrm{~mm}$.

\section{CRACK PATH STABILITY UNDER BIAXIAL LOADING}

The question of the stability of a crack path had been of interest for some time [8] but in general it wasn't possible to predict crack paths under biaxial fatigue loading. Therefore, in 1974 some tests [9], were carried out at room temperature on Waspaloy, a nickel based gas turbine material, in order to determine the conditions under which a fatigue crack path became unstable under biaxial loading. The specimens were $254 \mathrm{~mm}$ square and $2.6 \mathrm{~mm}$ thick. The material had been cross rolled during production to ensure that its properties were reasonably isotropic. Tests were carried out using sinusoidal constant amplitude loading at a stress ratio (ratio of minimum to maximum load in fatigue cycle), $R$, of 0.1 . In each test the fatigue load perpendicular to the crack was kept constant. Cracks were first grown from each end of an initial slit under uniaxial loading An in phase load was then applied parallel to the crack, and crack path behaviour observed. Figure 6 shows the crack path for a load parallel to the crack of twice the load perpendicular to the crack. The crack path became unstable and deviated from its initial path as soon as the load parallel to the crack was applied. At the time the tests were carried out it wasn't possible to do more than describe the results. However, reanalysis of these and other results in $1997[1,10]$ showed it was possible to correlate crack path stability in terms of a parameter called the $T$-stress ratio.

\section{PLASTIC DOMESTIC TAP}

In 1991 a plastic domestic tap in the author's utility room was observed to be leaking where it was screwed into a fitting on the supply pipe. The tap had a fitting for a hose pipe, and appeared to be a replacement for the original brass tap. When an attempt was made to unscrew the tap it failed completely. The two parts of the broken tap are shown in Figure 7 and a close up of the fracture surface in Figure 8. The dark area is fatigue and the light area the final static failure. The age of the tap at the time of failure is unknown, but as one fatigue cycle is applied each time a tap is turned on and off it is likely that thousands of cycles had been applied. Safety critical pressure containing components are often designed to leak-before-break [11] in order to avoid catastrophic failure. It is fortunate that the tap did so otherwise the utility room would probably have been flooded. The failed tap was replaced with a brass tap, and it was observed that the detail design in the vicinity of the threads was exactly the same. The replacement tap is still in use. The episode is an example of the danger of using a different material for a component without making appropriate changes to detail design.

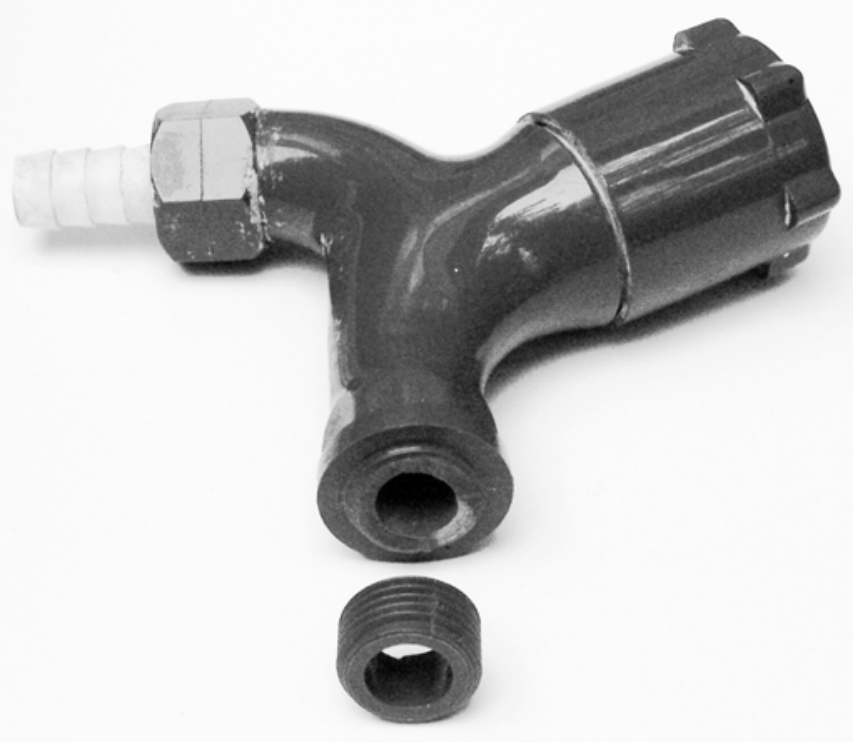

Figure 7. Plastic domestic tap. 


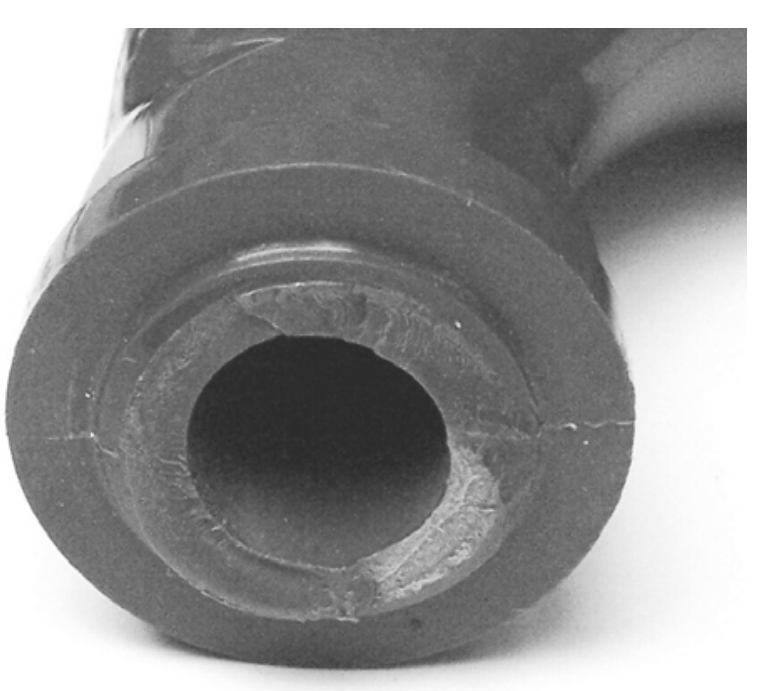

Figure 8. Crack surface of plastic domestic tap.

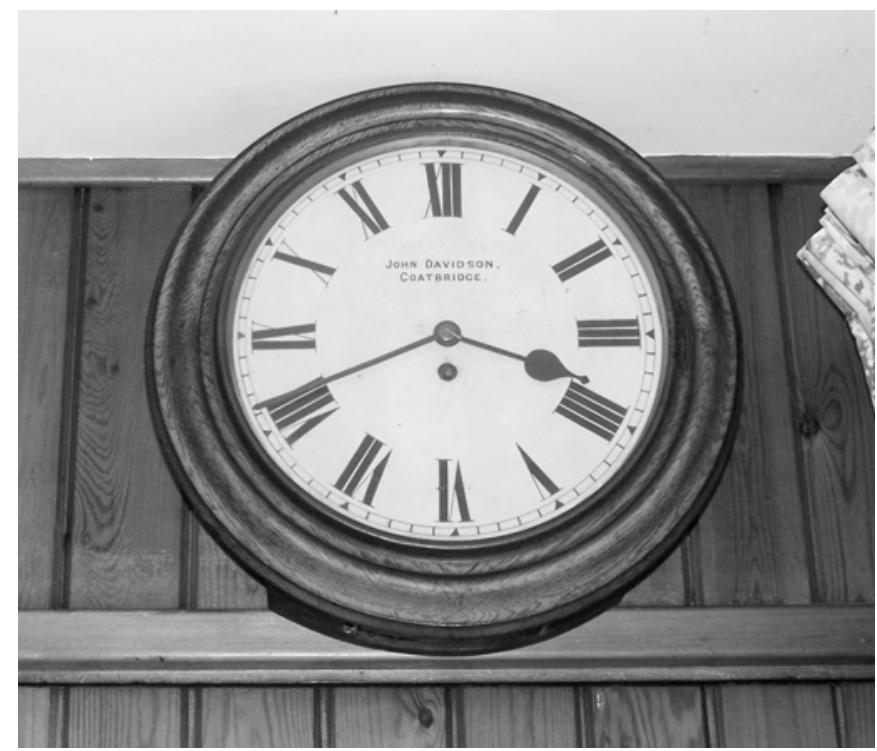

Figure 9. Wall clock by John Davidson, Coatbridge.

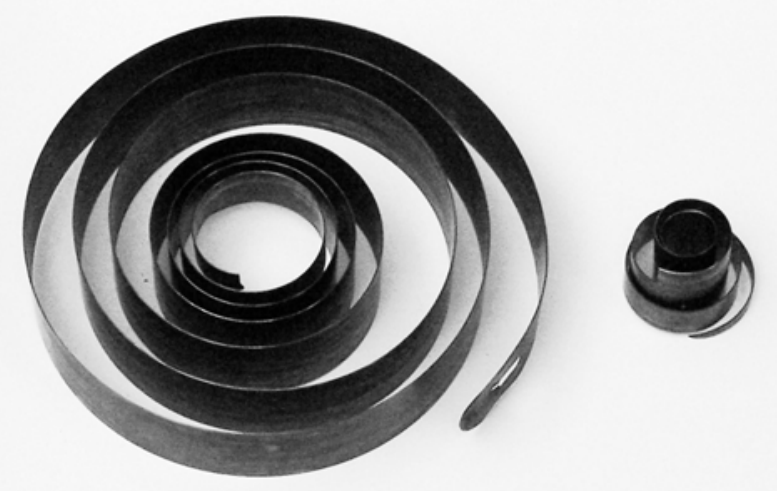

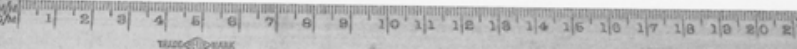

Figure 10. Failed wall clock mainspring.

\section{WALL CLOCK MAINSPRING}

Before the days of quartz clocks, spring driven wall clocks were widely used in public buildings. The exam- ple shown in Figure 9 was originally used in a school, but since 1968 it has been in use in the author's kitchen. In 1994 the mainspring failed while the clock was being wound. Examination showed that this was the final failure following fatigue crack growth. A general view of the failed mainspring is shown in Figure 10. Fatigue has been a problem in clock mainsprings for centuries, and traditionally they are designed using rules of thumb based on experience [12], rather than by detailed analysis. The total fatigue life is not known, but the clock is wound weekly so it must be thousands of cycles.

A clock mainspring is loaded in bending, with loading and unloading moving along the spring as it is wound and unwinds. When a mainspring breaks in fatigue the crack is usually straight across the spring, with crack growth predominantly through the thickness. However, in this particular mainspring crack path behaviour is unusually complicated, and details are shown in Figure 11. A fatigue crack initiated at a corner at one edge of the $27 \mathrm{~mm}$ wide mainspring. Initially, crack growth was across the spring (downwards in the picture) but after about $9 \mathrm{~mm}$ of growth the crack turned sharply towards the outer end of the spring (right in the picture), and then grew in a spiral fashion towards the other edge of the spring until the final failure took place. During this crack growth two secondary cracks initiated, and then joined so that a small triangular piece of spring became detached. The joined secondary crack then grew in a spiral fashion towards the centre of the spring, but did not contribute to the final failure. This is an example of a nuisance fatigue failure which did not have serious consequences. Such failures are not normally investigated at all. The offending component is simply replaced. In this particular case the replacement mainspring is still intact after 12 years.
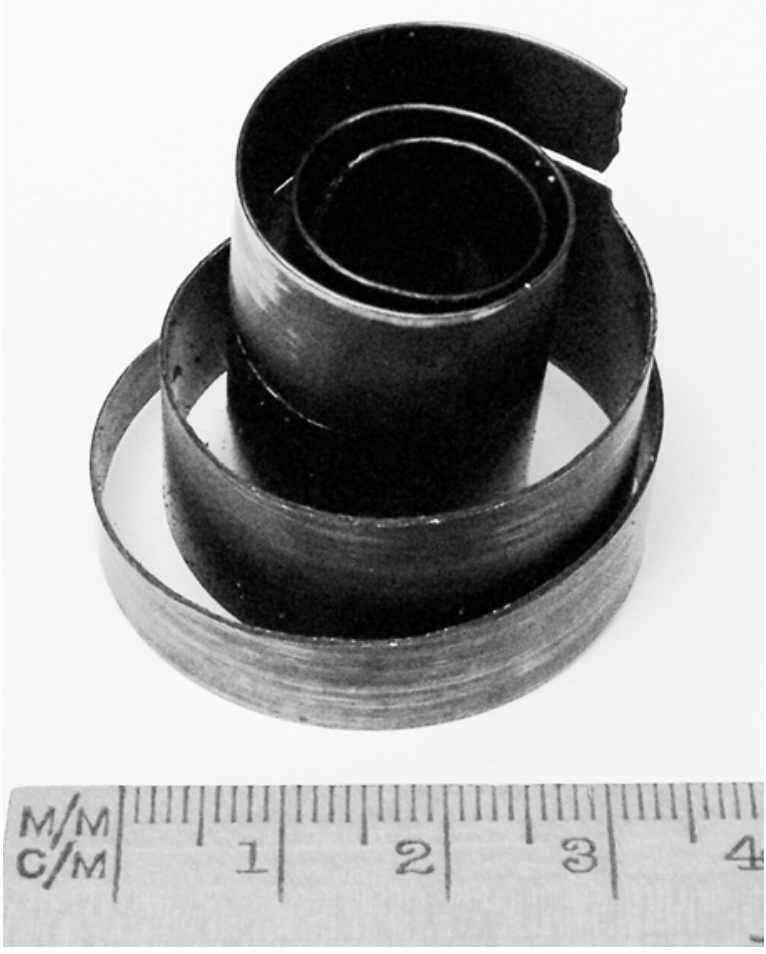

Figure 11. Centre portion of failed wall clock mainspring. 


\section{ANGLE NOTCH CHARPY SPECIMENS}

Some preliminary tests [13] were carried out in 1971 on angle notch Charpy specimens, but crack paths were not investigated in detail. Specimen design was based on the standard Charpy V-notch specimen with $\beta$ values (Figure 3) of $90^{\circ}$ (standard specimen), $75^{\circ}, 60^{\circ}$, and $45^{\circ}$. The true notch tip radius was reduced so that the notch tip radius measured in a plane parallel to the specimen sides was the same as in the standard Charpy specimen $(0.25 \mathrm{~mm})$. Figure 12 shows the appearance of specimens tested at 10 C. More detailed tests were carried out in 1997 using EN6a mild steel $(0.36 \% \mathrm{C})$ specimens [14]. All specimens were tested in the normalised condition (tensile strength $550 \mathrm{MPa}$, yield stress $280 \mathrm{MPa}$ ). Tests were carried out in a $300 \mathrm{~J}$ Charpy machine equipped with a 2 $\mathrm{mm}$ radius striker. They are an example of the complexity often observed in crack path behaviour under dynamic loading. The fracture surface appearance of the standard Charpy specimens $\left(\beta=90^{\circ}\right)$ is typical of mild steel. In the lower shelf region, that is at below about $-15^{\circ} \mathrm{C}$, fracture surfaces are crystalline, and in the upper shelf region, above about $30^{\circ} \mathrm{C}$, they are ductile. In the transition region fracture surfaces are initially ductile, and the amount of crystalline crack growth decreases with increasing temperature. Shear lips appear at above about $-15^{\circ} \mathrm{C}$, and increase in size with increasing temperature. The fracture appearance transition temperature (50 per cent crystalline) is about $25^{\circ} \mathrm{C}$. In the upper shelf region fracture surfaces are ductile.
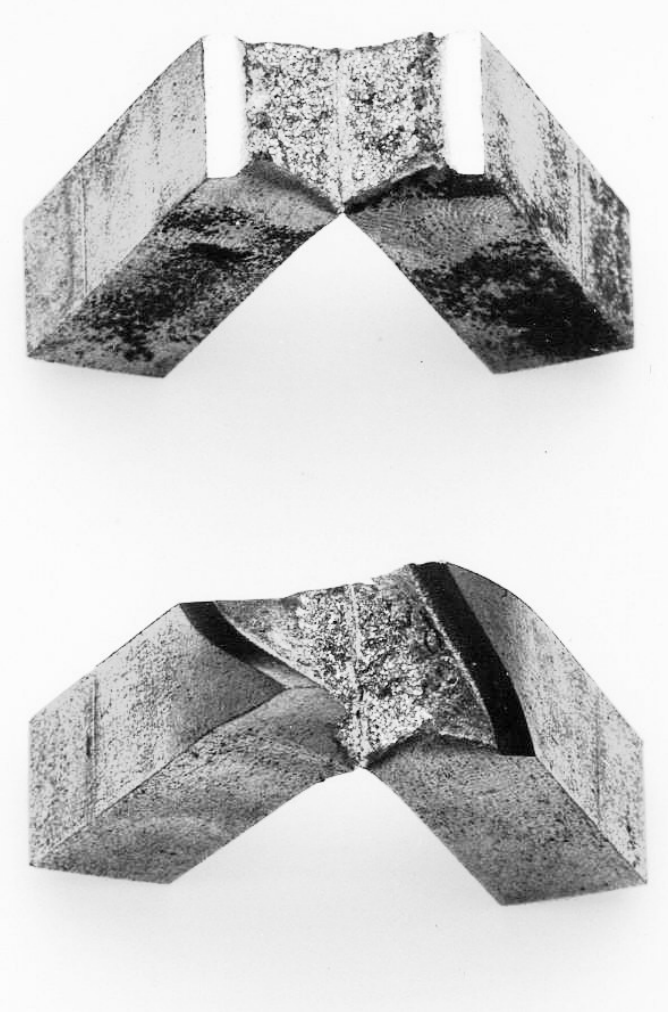

Figure 12. Fracture appearance of mild steel Charpy specimens tested at $10 \mathrm{C}$. Top, standard specimen, $\beta=90^{\circ}$. Bottom, angle notch specimen, $\beta=45^{\circ}$.
The fracture surface appearance of the angle notch specimens is controlled by a tendency towards square (Mode I) crack growth, but modified by plasticity and by crack path constraint due to the initial notch. The value of $\beta$ has little effect on either the 50 per cent crystalline transition temperature, or on the temperature below which fractures are crystalline. Shear lips for $\beta=75^{\circ}$ and $60^{\circ}$ are similar to those on standard Charpy specimens, but could not be distinguished for $\beta=45^{\circ}$. In the transition region fracture surfaces are initially ductile. The amount of initial ductile crack growth increases with increasing temperature. Crack initiation is along the notch tip, and in the notch plane, so the initial crack growth is mixed mode. For $\beta=$ $75^{\circ}$ and $60^{\circ}$ a crack twists as it grows, becoming Mode I as it approaches the striker position (Figure 3). For $\beta=$ $45^{\circ}$ there is an abrupt transition to Mode I crack growth (Figure 13).

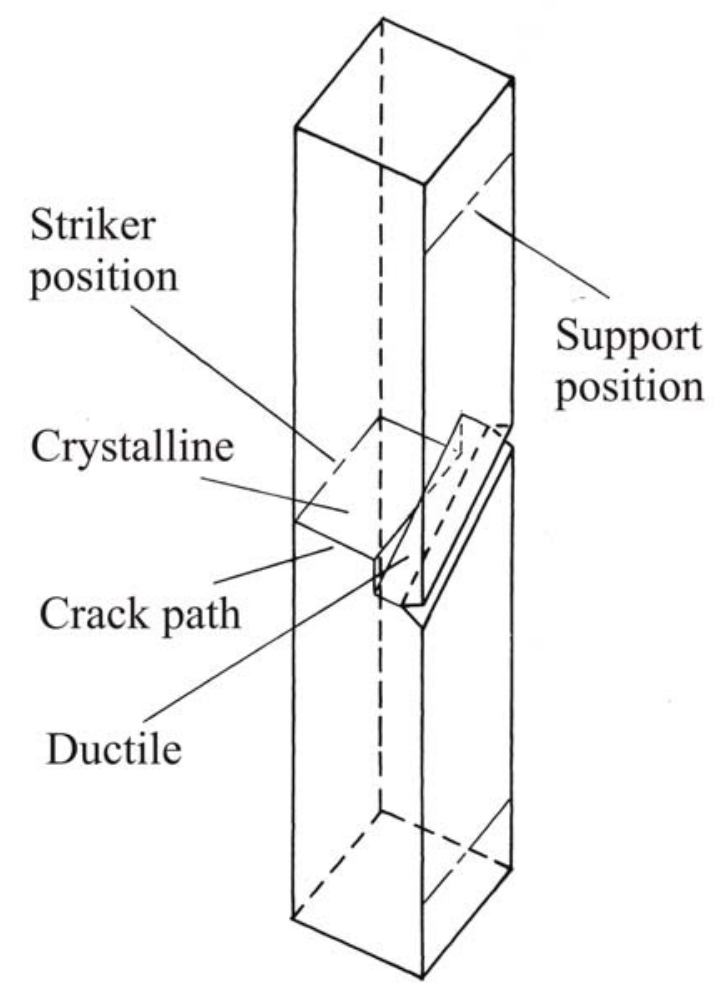

Figure 13. Angle notch Charpy specimen, abrupt transition to crystalline crack growth.

This Mode I growth is at least initially crystalline. At below about $-15^{\circ} \mathrm{C}$ fracture surfaces of the angle notch specimens are fully crystalline. Crack origins are Mode I. For $\beta=75^{\circ}$ and $60^{\circ}$ there are a number of individual Mode I crack origins along a notch tip, linked by vertical cliffs (apparently Mode III). The initial Mode I cracks link up as a crack grows, and overall a crack twists as it approaches the striker position. For $\beta=45^{\circ}$ the tendency to Mode I crack growth is so marked that the crack path is not constrained by the notch. At intermediate absorbed energy levels there is one crack origin at the centre of a notch, and crack growth is Mode I throughout (Figure 
14). At high absorbed energy levels there are crack origins at both notch corners. The cracks follow curved, apparently Mode I paths, as shown schematically for a single crack in Figure 15. The two paths merge as they approach the striker position.

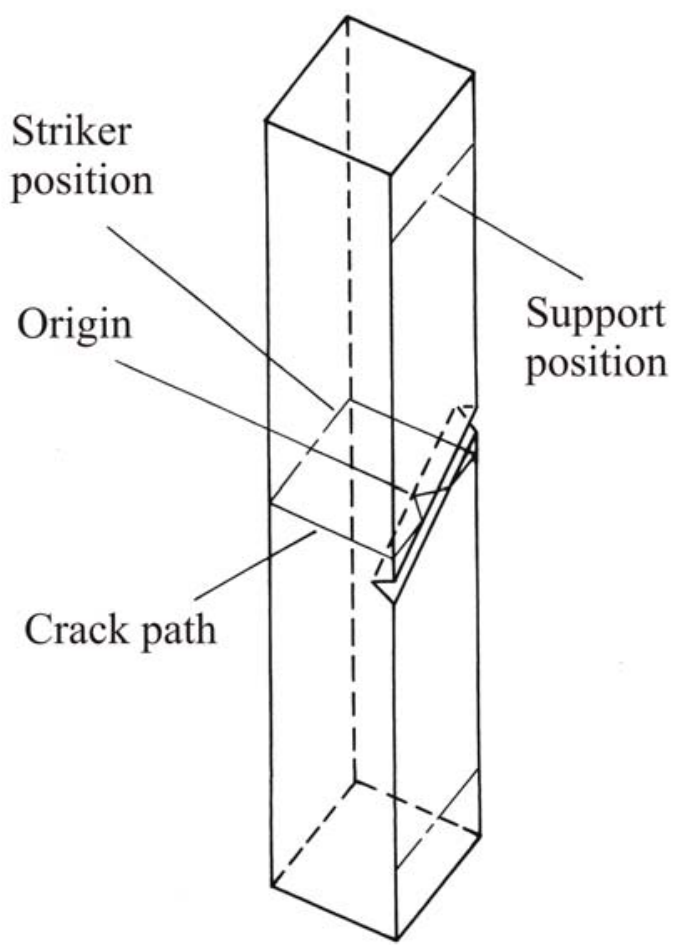

Figure 14. Angle notch Charpy specimen, crack origin at centre of notch.

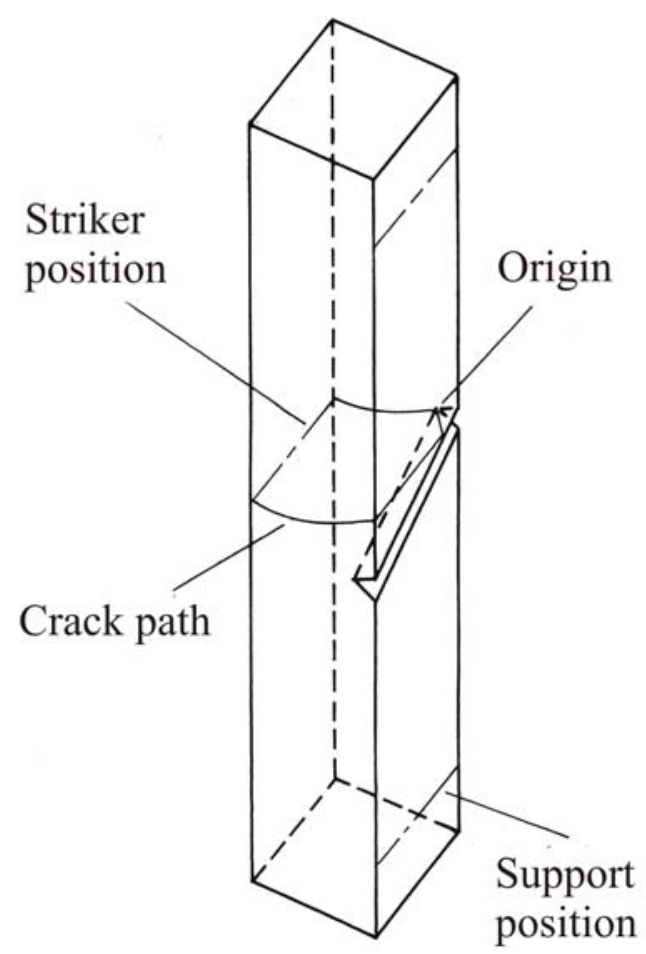

Figure 15. Angle notch Charpy specimen, crack origin at notch corner.

\section{CENTRAL HEATING BOILER BURNER}

During routine maintenance in 2002 one of the two burners in the gas fired domestic central heating boiler installed in the author's house was found to be cracked due to thermal fatigue. A general view of the burner is shown in Figure 16, and the crack is shown in Figure 17. The boiler was about 12 years old so, assuming it fired about 10 times per day, about 44,000 thermal fatigue cycles had been applied. The burner consists of a steel box with a series of small and large holes on top to distribute the gas to the flame above the box. The larger holes have reinforced perimeters. An internal wire mesh, just visible in Figure 17 , helps to distribute the gas evenly. Cracking appears to have initiated at three places on the perimeter of a smaller hole, grown into two larger holes with a small triangular piece becoming detached, and then two cracks grew across most of the width of the box, resulting in improper combustion. The designer did not appear to have appreciated the point that stress concentration factors are largely independent of hole size. The reinforcement had prevented crack initiation at the large holes but its absence had allowed cracking at a small hole. This is another example of a nuisance fatigue failure. Annual inspection was recommended by the boiler manufacturer. This ensured that the cracking was detected before it became dangerous, and the burner was replaced.

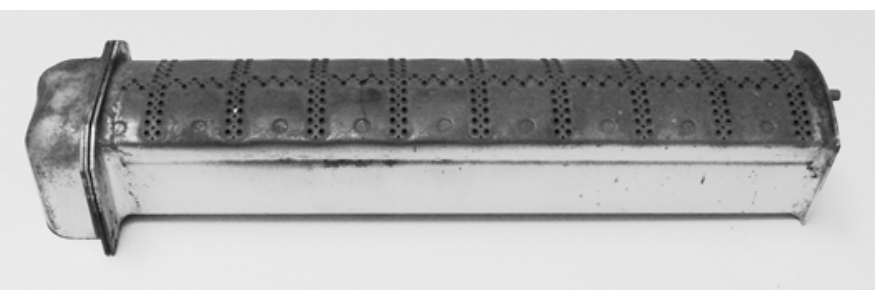

Figure 16. Burner from domestic central heating boiler.

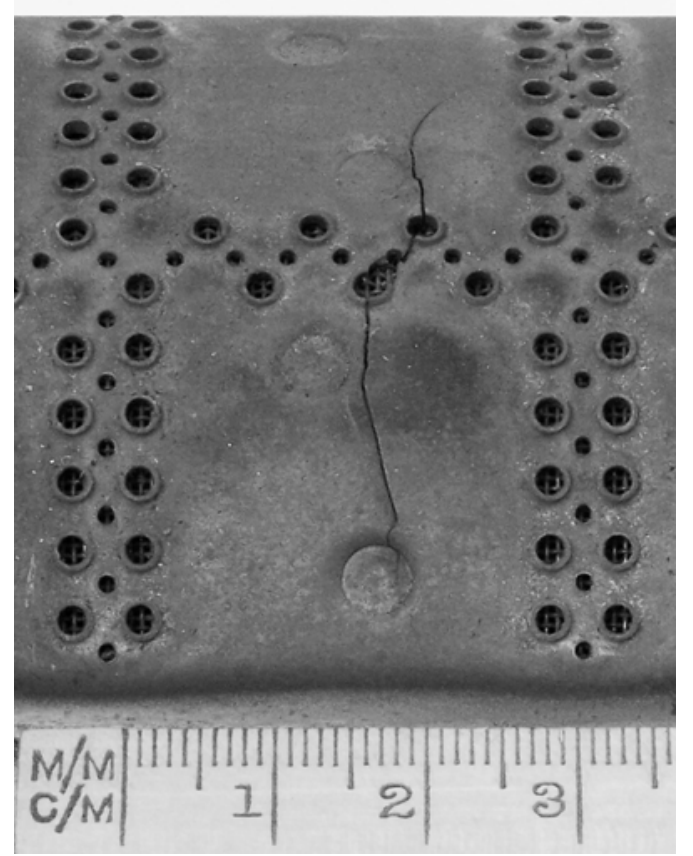

Figure 17. Crack in burner from domestic central heating boiler. 


\section{WALKING SHOE}

In 2005 the author found that the plastic soles of pair of walking shoes had become badly cracked and one no longer fitted properly. This more severely damaged shoe is shown in Figure 18. The sole of a shoe is subjected to repeated bending. Going uphill a sole is also subjected to repeated tension as the rearward force applied by the wearer's heel is transferred to the ground. This particular pair of shoes had covered several hundred kilometres, which is equivalent to around $3 \times 10^{5}$ cycles. In the shoe shown two separate cracks had initiated in grooves near the toe, grown past each other and then curved together, in a well known crack path behaviour [15], so that a piece of sole became detached. The heel had also cracked and, in what appears to have been the final event that reduced the stiffness of the shoe so much that it became unusable, the sole separated from the upper at the end of this crack. The use of a plastic, instead of rubber, for the soles has reduced the rate of wear but led to fatigue failure. This is another example where a change of material has resulted in fatigue cracking.

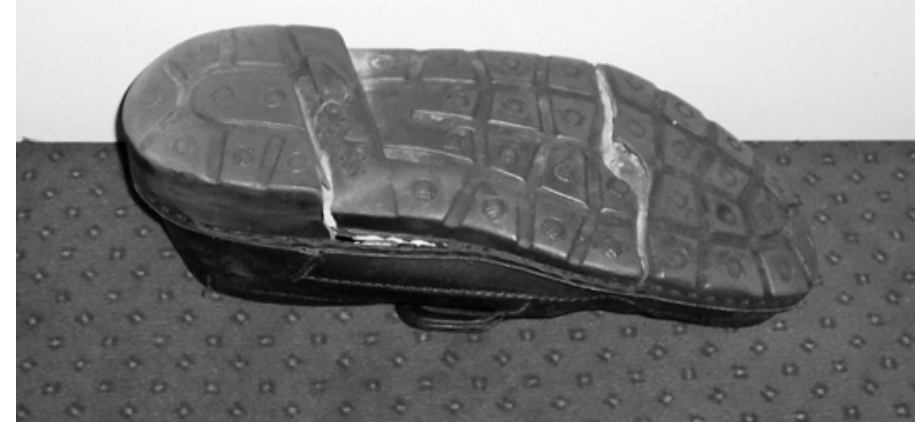

Figure 18. Cracks in sole of walking shoe.

\section{CONCLUDING REMARKS}

Paths taken by cracks have been of interest for a very long time. A large amount of empirical knowledge has been accumulated, but at the present state of the art the factors controlling the path taken by a crack are not completely understood. The numerous possible crack configurations [7] mean that a systematic approach to the determination of crack paths isn't feasible, so particular practical problems need to be tackled on an ad hoc basis. The examples given have been chosen from the author's experience to illustrate the variety of crack paths which occur in practice.

\section{REFERENCES}

[1] L. P. Pook, Crack Paths, WIT Press, Southampton (2002).

[2] R. Cazaud, Fatigue of metals, Chapman \& Hall Ltd, London (1953).
[3] J. Longson, A photographic study of the origin and development of fatigue fractures in aircraft structures. RAE Report No. Struct 267. Royal Aircraft Establishment, Farnborough (1961).

[4] J. E Srawley, W. F. Brown, Fracture toughness testing methods. In Fracture toughness testing and its applications. ASTM STP 381. American Society for Testing and Materials, Philadelphia, PA, (1965) 133.

[5] L. P. Pook, Brittle Fracture of Structural Materials Having a High Strength Weight Ratio. PhD thesis, University of Strathclyde, Glasgow (1968).

[6] L. P. Pook, Eng. fract. Mech., 3 (1971) 205.

[7] L. P. Pook, D. G. Crawford, The fatigue crack direction and threshold behaviour of a medium strength structural steel under mixed Mode I and III loading. In: Kussmaul, K., McDiarmid, D. L. and Socie, D. F. (Ed). Fatigue Under Biaxial and Multiaxial Loading. ESIS 10. (1991) 199. Mechanical Engineering Publications, London.

[8] B. Cotterell, Int. J. fract. Mech., 2 (1966) 526.

[9] L. P. Pook, R.Holmes, In: Proc. Fatigue Testing and Design Conf., Society of Environmental Engineers Fatigue Group, Buntingford, Herts, 2 (1976) 36.1

[10] L. P. Pook, An alternative crack path stability parameter. In: Brown, M. W., de los Rios, E. R. and Miller, K. J. (Eds). Fracture from Defects. ECF 12. EMAS Publishing, Cradley Heath, West Midlands. I (1998) 187.

[11] L. P. Pook Linear Elastic Fracture Mechanics for Engineers. Theory and Applications. WIT Press, Southampton (2000).

[12] F. J. Britten, The watch \& clock makers' handbook, dictionary and guide. $16^{\text {th }}$ Edition. Revised by Good, R. Arco Publishing Company Inc, New York (1978)

[13] L. P. Pook, Eng. fract. Mech., (1972) 483.

[14] L. P. Pook, M. J. Podbury, Int. J. Fract., 90, (1998) L3-L8.

[15] S.Melin, Int. J. Fract., 23(1) (1983) 37.

[16] L.P. Pook, Keyword Scheme for a Computer Based Bibliography of Stress Intensity Factor Solutions. NEL Report 704. National Engineering Laboratory, East Kilbride, Glasgow (1986). 